\title{
RESPONSE OF WATER CHERENKOV DETECTORS TO SMALL SIGNALS: A CASE STUDY
}

\author{
NGUYEN THI THAO, PHAM NGOC DIEP, PHAM NGOC DONG, \\ PHAM THI TUYET NHUNG, PIERRE DARRIULAT, \\ PHAM TUAN ANH AND DO THI HOAI \\ VATLY, Institute for Nuclear Science and Technology, \\ 179 Hoang Quoc Viet, Nghia Do, Cau Giay, Hanoi, Vietnam \\ Received 17 April 2013 \\ Accepted for publication 23 July 2013
}

\begin{abstract}
Detailed studies of the response of the VATLY water Cherenkov detector, a replica of those used in the ground array of the Pierre Auger Observatory, are presented with emphasis on the response to low amplitude signals. The method used is to detect decay electrons from muons stopping in the water volume. Results include properties of the detection process as well as of the atmospheric cosmic ray flux.
\end{abstract}

\section{INTRODUCTION}

For now nine years, the Pierre Auger Collaboration, with which our laboratory, VATLY, is associated, has been operating a giant ground array of Cherenkov detectors covering $50 \times 60 \mathrm{~km}^{2}$ in the Argentinean Pampas [1,2]. Its aim is the study of extragalactic Ultra High Energy Cosmic Rays, with energies in the $10^{20} \mathrm{eV}$ range. It has already given first evidences for a cut-off of the energy spectrum [3] corresponding to the photoproduction threshold on the Cosmic Microwave Background (GZK cut-off) and for a positive, but weak, correlation with nearby galaxies - in particular Centaurus A - as potential sources [4].

As a contribution to the work of the Pierre Auger Collaboration, we have assembled on the roof of our Hanoi laboratory a replica of one of the 1660 Cherenkov detectors of the Pierre Auger Observatory (PAO) with the aim of training and gaining familiarity with the tools and methods used at the PAO. Together with other equipment, including scintillator detectors and additional smaller Cherenkov detectors, it has given us an opportunity to explore some features of the cosmic ray flux in Hanoi where the rigidity cut-off reaches its world maximum of $17 \mathrm{GV}$ [5].

The present work covers detailed studies that have been made of the performance of the VATLY Cherenkov detector with emphasis on its response to low signals. The detector (Fig. 1) is a water cylinder, $10 \mathrm{~m}^{2}$ in area and $1.2 \mathrm{~m}$ in height, equipped with three down-looking 9" Photo Multiplier Tubes (PMTs). In the PAO regime, where the detectors sample $\sim 5 \mathrm{ppm}$ of the $\mathrm{PAO}$ area, one deals with signals reaching $10^{3} \mathrm{VEM}$, a VEM - Vertical Equivalent Muon - being the signal produced by a vertical relativistic 
muon impacting a detector in its centre. Here, we explore the response down to a tenth of a VEM, implying a dynamical range in excess of $10^{4}$. Such a large dynamical range is important to obtain accurate measurements of the Lateral Distribution Function (LDF) and, consequently, of the shower energy. It is limited by saturation at high signal amplitudes, which is taken care of by recording the raw anode signal together with the amplified dynode signal of each PMT. Its behaviour at low signal amplitudes is the main objective of the present study.

\section{EXPERIMENTAL METHOD}

The method that we have been using to study low amplitude signals is to look for decays of muons stopping in the water volume of the Cherenkov detector. Only a small fraction of cosmic muons, typically 6 to $7 \%$, do stop in there and of these, an even smaller fraction produces sufficient Cherenkov light to be detected before stopping (at the scale of a few tenths of a VEM). The subsequent muon decays occur on average some two microseconds afterward, producing an electron (or positron) and a neutrino-antineutrino pair that escapes the water volume undetected. The electron carries an average energy of only $\sim 35$ $\mathrm{MeV}$, producing a signal of only a fraction of a VEM in ideal detecting conditions. Our experimental set-up has been designed to study such decays by detecting the signals produced by both the stopping muon and the decay electron. Such pairs have been detected using different thresholds and delays, and the amplitude of the second signal has been recorded together with the time separating the two signals. Such data make it possible, using the different time dependences, to disentangle the contribution of muon decays from that of random muon coincidences.

In addition to the main Cherenkov detector, we have assembled a scintillator hodoscope bracketing it from above and below (Fig. 1) that provides a trigger on central relativistic feed-through muons for calibration purpose. The results of the calibration are displayed in Fig. 2. The width (rms) to mean ratio in the Cherenkov case is $26 \%$, corresponding to $\sim 14$ photoelectrons per VEM.

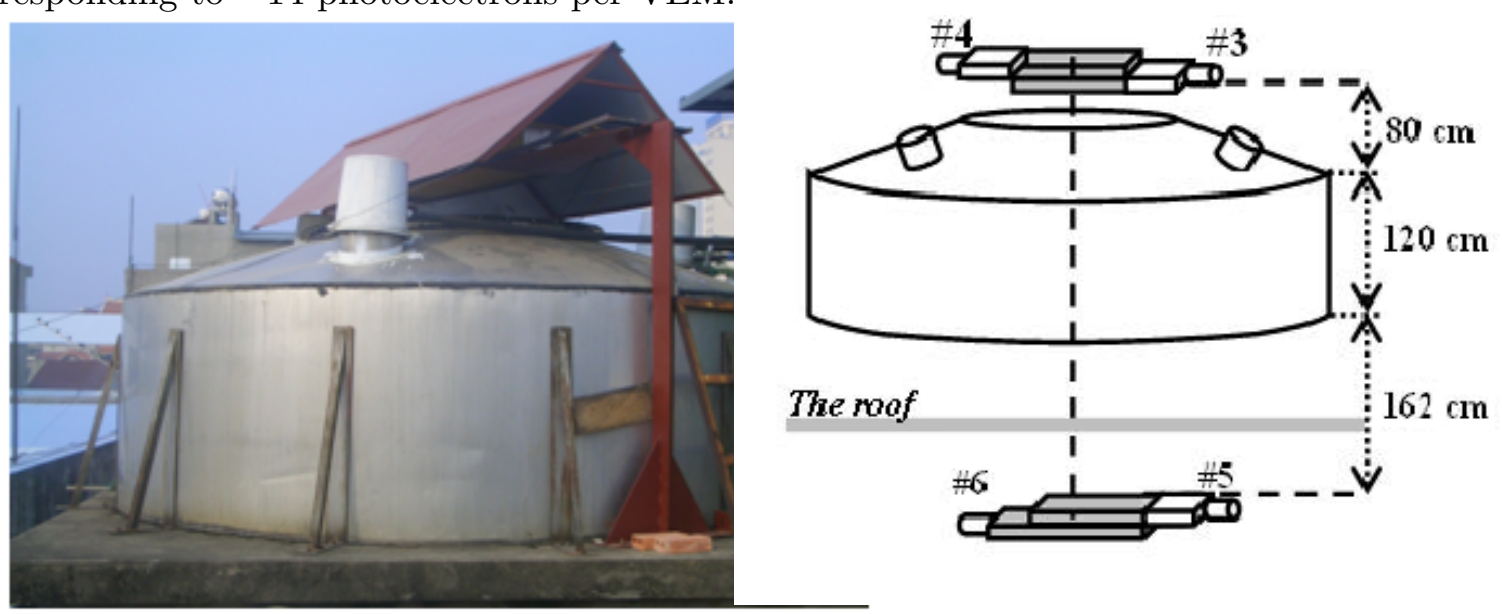

Fig. 1. Left: Photograph of the VATLY Cherenkov tank and the upper hodoscope. Right: Geometry of the whole assembly. 

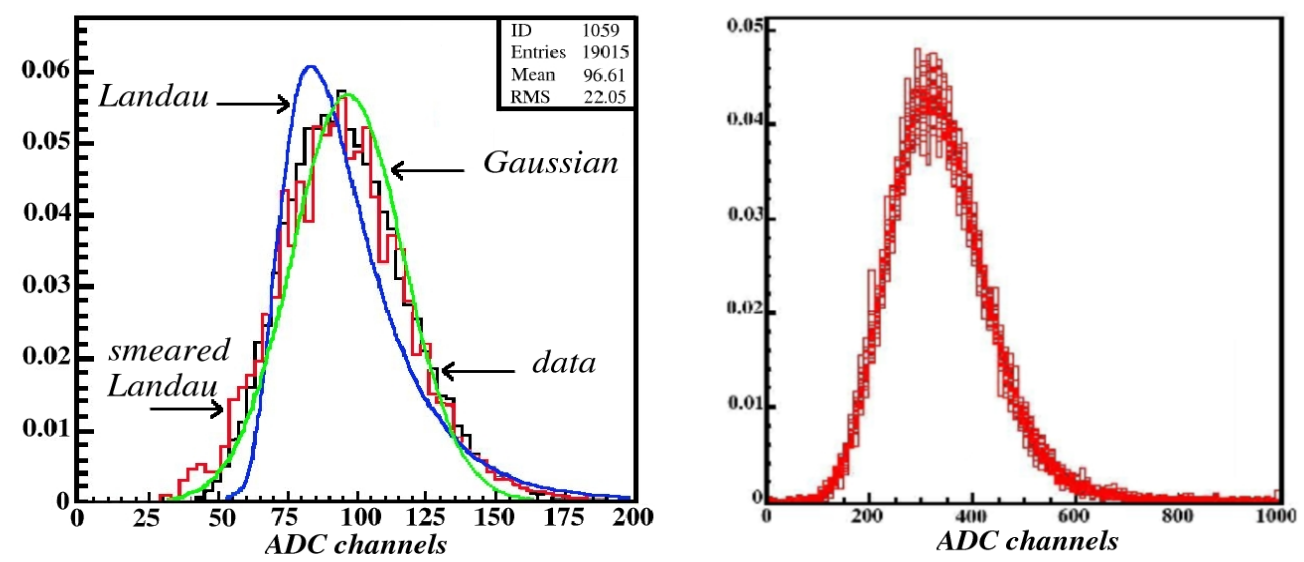

Fig. 2. Left: mean charge distribution of the four hodoscope scintillator signals with a Landau fit, a Gaussian fit and a smeared Landau fit. Right: mean charge distribution of the two Cherenkov PMTs used to produce the main signal. Curves measured in different runs have been superimposed.

The basic processes of relevance are the slowing down of muons in water due to ionization energy losses and the emission of Cherenkov photons. The largest possible track length in the Cherenkov tank is $l_{\max }=\sqrt{(1.2)^{2}+(3.6)^{2}} \sim 3.8 \mathrm{~m}$. Such a range corresponds to a muon kinetic energy $T \sim 0.8 \mathrm{GeV}$. The differential energy loss is therefore close to minimum $\left(d T / d x=1.8 \mathrm{MeVg}^{-1} \mathrm{~cm}^{2}\right.$ at $\left.T=331 \mathrm{MeV}\right)$ or, in the lower energy range, inversely proportional to the muon kinetic energy [6]. The track length $l$ of muons in water increases as a function of kinetic energy $T$ as shown in Fig. 3 (left); vertical muons start to feed through the tank $(l>1.2 \mathrm{~m})$ as soon as $T$ exceeds $280 \mathrm{MeV}$.

The second basic process of relevance is the emission of Cherenkov radiation. The Cherenkov threshold is $\beta_{0}=1 / n=0.75$ where $n=1.34$ is the refractive index of water. This corresponds to a kinetic energy, $T_{0}=\left(E_{0}-m\right)=54 \mathrm{MeV}$, where $m=106 \mathrm{MeV}(c=1)$ is the muon rest mass, and $E_{0}=\gamma_{0} m=m / \sqrt{1-\beta_{0}{ }^{2}}$. The half-aperture of the Cherenkov cone is $\theta=\cos ^{-1}(1 / \beta n)$ and the density $d N / d x$ of Cherenkov photons radiated by a muon having velocity $\beta$ over a distance $d x$ is proportional to $\sin ^{2} \theta=1-1 /(\beta n)^{2}$. For $\beta=1, \theta \sim 41^{\circ}$. It is convenient to use VEM as unit: 1 VEM $\sim 120 \times 80 \times\left(1-1 / n^{2}\right) \sim 4.210^{3}$ photons, where the number of Cherenkov photons per centimetre has been taken equal to 80 in the wave length range of relevance. With three PMTs and a photocathode efficiency of $\sim 10 \%$, this means 140 photoelectrons per VEM for a perfect optical cavity, compared with $\sim 100$ in the PAO. Hence, in VEM units, independently from the number of Cherenkov photons radiated per centimetre,

$d N / d x=\left(1-1 /(\beta n)^{2}\right) /\left(1-1 / n^{2}\right) / 120=1.910^{-2}\left(1-1 /(\beta n)^{2}\right) \mathrm{VEMcm}^{-1}$.

A muon having a low enough kinetic energy stops in the water tank. The number of photons it radiates depends only on the kinetic energy it has when entering the tank and so do its track length and the track length over which it radiated photons. Fig. 3 (right) shows the dependence on kinetic energy of the total number of Cherenkov photons radiated by a muon before stopping. 

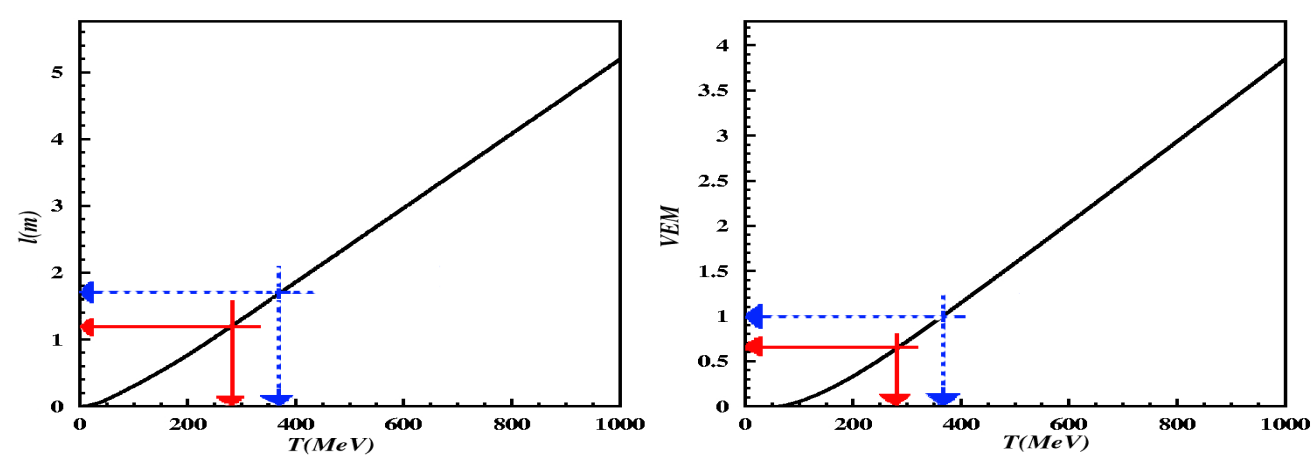

Fig. 3. Dependence on muon kinetic energy (MeV) of track length (m, left panel) and of the number of Cherenkov photons (VEM, right panel). The full arrows are for a muon that just stops in $1.2 \mathrm{~m}$ of water and the dotted arrows for a muon that just emits 1 VEM before stopping.

It is straightforward to estimate what to expect in terms of rates. For this purpose, muons having a mean kinetic energy of $4 \mathrm{GeV}$ and the proper angular distribution [5] are generated at a distance from the detector centre uniformly distributed between zero and $l_{\max }$. It is useful to distinguish between four families of trajectories (Fig. 4, left): 1, missing the tank all together; 2 , crossing the upper or lower plate and the side wall; 3 , crossing both the upper and lower plates; 4, crossing twice the side wall. A muon may produce no detectable photon either because it misses the tank or because its kinetic energy is lower than the Cherenkov threshold. Moreover, it may or may not stop in the tank. If it does not, it does not produce any detectable decay electron. Table 1 gives the properties of the four families and Fig. 4 (right) displays the distributions of the track length, to which the Cherenkov light emitted by feed-through muons (in VEM) is proportional. While the plate-wall and plate-plate configurations have comparable abundances, the wall-wall configuration is much less likely, only $\sim 1 \%$ of the total.
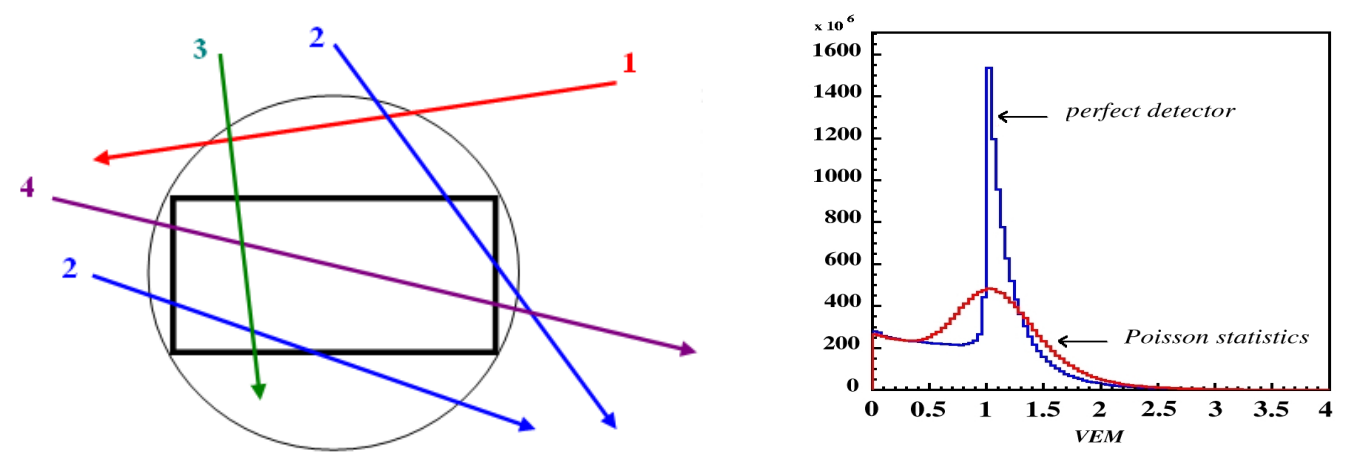

Fig. 4. Left: Definition of trajectory families for feed-through muons. Right: Distribution of the charge (VEM) deposited by feed-through muons either assuming a perfect detector or Poisson statistics using 14 photoelectrons per VEM. 
Table 1. Properties of the four families of trajectories.

\begin{tabular}{|c|c|c|c|c|}
\hline Family & $\begin{array}{c}1 \\
\text { Missing }\end{array}$ & $\begin{array}{c}2 \\
\text { Wall-Plate }\end{array}$ & $\begin{array}{c}3 \\
\text { Plate-Plate }\end{array}$ & $\begin{array}{c}4 \\
\text { Wall-Wall }\end{array}$ \\
\hline Abundance & $9.7 \%$ & $42.2 \%$ & $47 \%$ & $1.1 \%$ \\
\hline Fraction stopping & - & $5.3 \%$ & $7.8 \%$ & $9.2 \%$ \\
\hline$<$ VEM $>$ feed-through & - & 0.73 & 1.19 & 1.47 \\
\hline$<$ VEM $>$ stop & - & 0.22 & 0.28 & 0.55 \\
\hline
\end{tabular}

The predicted fraction of stopping muons is $\sim 6.5 \%$ on average, slightly larger for the plate-plate configuration than for the plate-wall configuration. On average, the Cherenkov light emitted by a stopping muon corresponds to one quarter of a VEM. The expected total rate is $\sim 1.4 \mathrm{kHz}$ for feed-through muons and therefore $\sim 20 \mathrm{~Hz}$ for coincidences of two feed-through muons in a $10 \mu \mathrm{s}$ window. The rate of pairs with a stopping muon in second position is therefore $\sim 1.3 \mathrm{~Hz}$. Of these, only a fraction will be detected and an even smaller fraction will produce a detectable decay electron.

For the decay electron (meaning electron or positron) to be detected, the muon should not stop too close from the tank walls: when calculating the expected rate, one needs to account for the incomplete shower containment in such cases. Once averaged over muon polarization, the electron may be emitted forward or backward with equal probabilities and the bulk of the electron shower covers some 20 to $40 \mathrm{~cm}$ (one radiation length is $36 \mathrm{~cm}$ ). The kinetic energy $T$ carried by the decay electron, averaged over polarization and electron emission angle, has a distribution of the form $d N / d x=2\left(3 x^{2}-2 x^{3}\right)$ where $x=2 T / m$ varies between 0 and 1 . The mean value of $x$ is 0.7 , meaning for $T$ a mean value of $37 \mathrm{MeV}$.

\section{EXPERIMENTAL SET-UP}

The recorded data include time autocorrelation spectra and charge distributions. The autocorrelation spectra use a Time-to-Amplitude Converter (TAC), the output of which is sent to a Multi-Channel Analyzer (MCA). The charge distributions are measured in Analogue-to-Digital Converters (ADC) using a CAMAC data acquisition system. A fast electronic circuit, using the NIM standard, is used as front end (Fig. 5). The signal studied is a coincidence between the amplified dynode signals of PMTs 1 and 2 of the Cherenkov detector. It is used as both start and stop of the TAC but care is taken to maintain a low rate at the TAC input in order to assure its proper behaviour. This is done by replacing the main signal by a coincidence between itself and another main signal occurring within a time window of $10 \mu$ s following it. With such logic, both the start and stop inputs of the TAC are activated only when the main signal is followed by another within a window $\left[D_{1}, D_{1}+10 \mu \mathrm{s}\right]$ with respect to the first, thus reducing the rate considerably. It must be noted, however, that it is the second of these signals that starts the $\mathrm{TAC}$ and the first that stops it, resulting in a time distribution inverted with respect to normal.

The response of the MCA to a time difference $\delta t$ between the two signals has been carefully calibrated using reference signals and including corrections for a small nonlinearity. From the calibration data, we obtain an estimate of the uncertainty on the 
time measurement of $60 \mathrm{~ns}$.

The charges of both the hodoscope scintillator signals and Cherenkov signals are recorded in a single 12-input ADC module activated by three possible gates. A first gate is generated by the TAC start signal, namely the second signal of a correlated pair; a second gate is generated by a clock and is used to measure pedestals; the third gate is generated by a coincidence between the four scintillator plates of the hodoscope. All three gates are converted to a common broad gate, an essential requirement in order to be able to have a common charge scale and to perform the VEM calibration properly. The timing of the gate with respect to the signals being analysed has been carefully adjusted to ensure that the signals are in all cases well contained within the gate. Pattern units are used to tell which trigger was active.

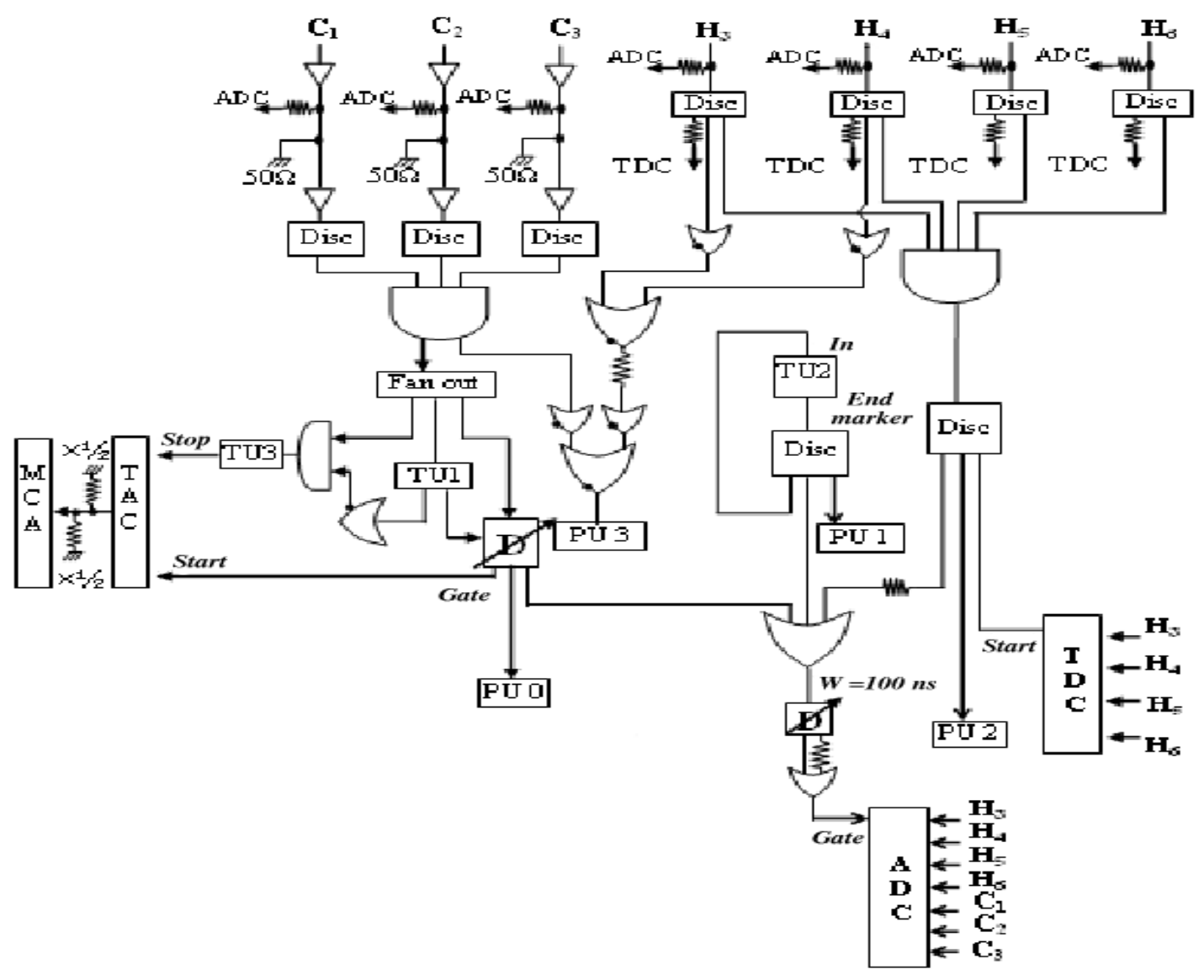

Fig. 5. Block diagram of the electronics. The three amplified Cherenkov signals $C_{i}$ and the four hodoscope signals $H_{i}$ are fed to ADCs and discriminators via passive splitters. Discriminator output signals are used to construct a Cherenkov trigger (tagged by pattern unit PU0) and a hodoscope trigger (tagged by pattern unit PU2). A clock (TU2) provides a trigger tagged by pattern unit PU1 used to measure ADC pedestals. 


\section{DATA COLLECTION AND ANALYSIS}

\section{IV.1. Time autocorrelation spectra}

A total of 62 time autocorrelation spectra have been collected. A number of crosschecks have been performed in order to ascertain the quality of the recorded data, including an independent evaluation of the width of the time window, $9.98 \pm 0.06 \mu \mathrm{s}$. The 62 spectra are fitted simultaneously to a sum of four exponentials that reads:

$$
R \exp (-R \delta t)+g_{0} R_{s h} \exp \left(-R_{s h} \delta t\right)+\varphi \rho_{+} R_{+} \exp \left(-R_{+} \delta t\right)+\varphi \rho_{-} R_{+} \exp \left(-R_{-} \delta t\right) .
$$

Here, $\rho_{+}=1-\rho_{-}$and $\rho_{-}$(the fractions of positive and negative muons), as well as $R$, the cosmic muon rate, $R_{+}$and $R_{-}$(the muon decay rate in vacuum and the disappearance rate of negative muons, including both decays and capture in water), are fixed to their known values. The fitted parameters are $g_{0}$ and $R_{s h}$, describing the multimuon contribution (two muons from a same air shower) and $\varphi$, describing the electron detection efficiency, equal to the product $f_{\text {stop }} \eta$ where $f_{\text {stop }}$, the fraction of stopping muons is the same for all runs, while $\eta$, the electron detection probability, depends on threshold. As seven different threshold values have been used, the total number of parameters to be adjusted is 9 .

The total number of events in each spectrum is normalized to a common value between data and model. The normalization constant is observed to be proportional to the duration of the measurement as expected. The $\chi^{2}$ of the fit is calculated using statistical errors exclusively. The results of the best fit are listed in Table 2 and illustrated in Fig. 6. The value of the best fit $\chi^{2}$ is 1.02 per degree of freedom (of which there are 118185), providing evidence for the quality of the fit and for negligible systematic errors. The measured electron contribution shows a very good agreement with the nominal muon life time. The dependence of $\varphi$ on threshold shows that the sensitivity to electron detection drops to zero when the threshold reaches its higher values.

Table 2. Time autocorrelation spectra: best fit results.

\begin{tabular}{|c|c|c|}
\hline$\chi^{2} / d o f$ & 1.02 & Threshold (threshold units) \\
\hline$g_{0}\left(10^{-5}\right)$ & $0.79 \pm 0.05$ & - \\
\hline$R_{s h} \mu s^{-1}$ & $0.89 \pm 0.03$ & - \\
\hline$\varphi_{1}(p p m)$ & $123 \pm 0.7$ & 0.5 \\
\hline$\varphi_{2}(p p m)$ & $88 \pm 0.6$ & 0.7 \\
\hline$\varphi_{3}(p p m)$ & $65 \pm 0.6$ & 1.0 \\
\hline$\varphi_{4}(p p m)$ & $19 \pm 0.5$ & 1.5 \\
\hline$\varphi_{5}(p p m)$ & $1.3 \pm 0.5$ & 2.0 \\
\hline$\varphi_{6}(p p m)$ & $<0.01$ & 2.5 \\
\hline$\varphi_{7}(p p m)$ & $<0.02$ & 3.0 \\
\hline
\end{tabular}

The best fit value of parameter $g_{0}$ is $(0.79 \pm 0.05) 10^{-5}$ for a decline time of $1.13 \pm 0.04$ $\mu s$, meaning a rate of $7.0 \pm 0.5 \mathrm{~Hz}$ compared with an inclusive muon rate of $\sim 2 \mathrm{kHz}$, namely a probability of $3.5 \%$ oto detect a second muon from the same shower when one has already been detected. For a muon multiplicity of 2 , it means a shower radius of $\sim 30$ $\mathrm{m}$, in agreement with typical sea-level decoherence functions. 

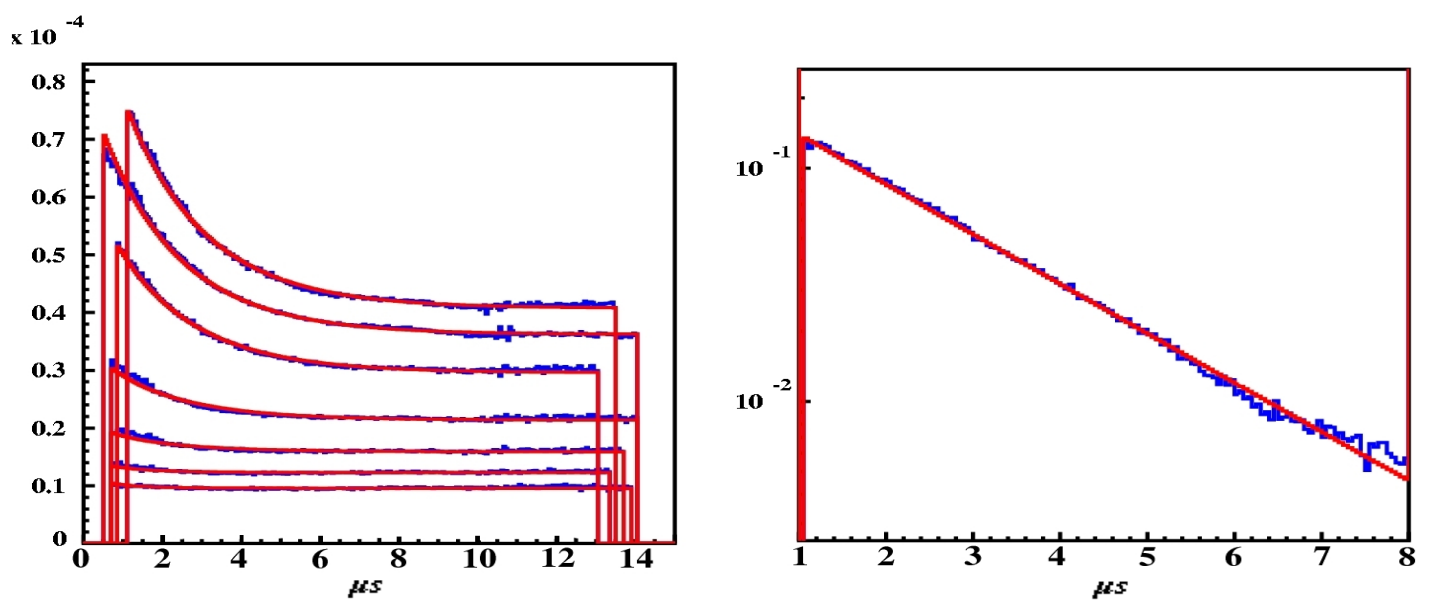

Fig. 6. Measured time distributions ( $\mu$ s, histograms) as compared with the result of the best fit (smooth curves). Left: Autocorrelation spectra for different thresholds. Right: Electron contribution from muon decays. In most bins data and fit are indistinguishable.

\section{IV.2. Charge distributions}

The second signal of a correlated pair, of which the charge is recorded in the ADC, is either an electron (requiring that the first signal was a detected stopping muon) or a muon (minimum ionising in most cases). Both components depend in different ways on threshold and delay.

A total of 42 runs have been retained for a simultaneous analysis after proper VEM calibration using hodoscope data. The charge distributions are fitted to a form $S_{i, j, k}=N_{i, j} C_{i}\left\{F_{k}^{\mu}+\lambda_{i} \exp \left(-D_{j} / \tau\right) F_{k}^{e l}\right\}$ where $i$ labels the threshold, $j$ the delay and $k$ the charge bin. $N_{i, j}$ is a normalisation constant, one for each spectrum; $\lambda_{i}$ accounts for the fact that when the threshold increases, the fraction of stopping muons decreases (the threshold applies to both the start and stop signals) and so does that of possibly detected decay electrons; the exponential term accounts for the exponential decrease of the electron contribution as a function of delay $D_{j}$ and $\tau$ is taken equal to $2.0 \mu \mathrm{s}$ in order to account for capture on average; $C_{i}$ describes the cut-off at low charges due to the discriminator threshold, $t h_{i}$, expressed in threshold units. Rather than fitting the normalisation constants for each independent charge distribution, we set it to unity and normalise the measured distributions in the high charge region where electrons do not contribute. The data normalised in this fashion are displayed in Fig. 7 and compared with the results of the best fit. While a qualitative agreement is generally obtained, the quality of the fit is not very good, revealing a number of imperfections of the model which are addressed below. 

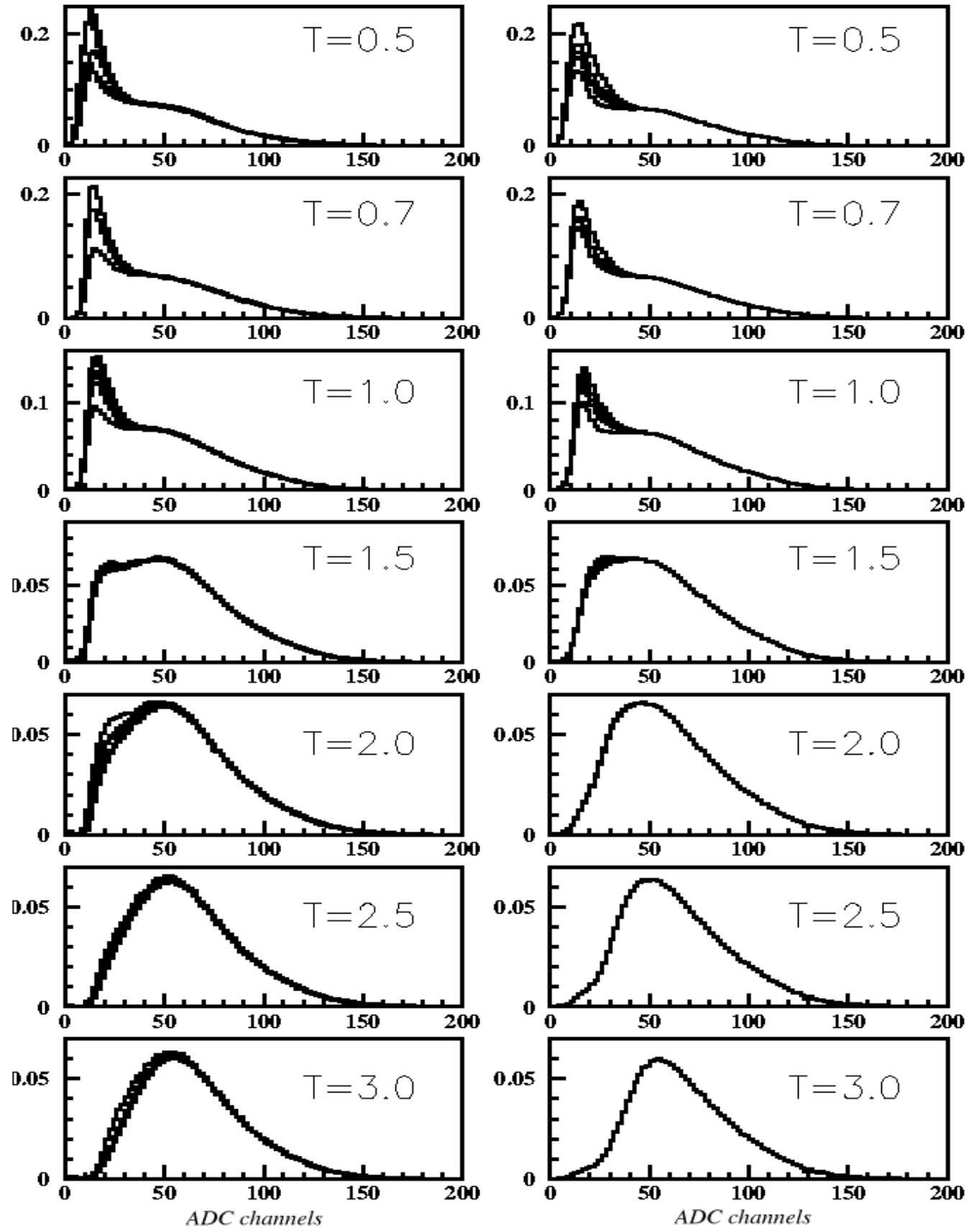

Fig. 7. Measured charge distributions (left panels) and best fit results (right panels). Each panel is labelled by the associated threshold value and includes curves taken at different delays. A number of imperfections in the fit quality are clearly visible. 
In particular, the cut-off is simply described by a function $C_{i}$ taken to switch from 0 to 1 around $x=a_{c} t h_{i}$ over a width measured by $b_{c} t h_{i}$, a form which will later be found inadequate. The best fit values of $a_{c}$ and $b_{c}$ are $0.18 \pm 0.02 \mathrm{VEM}$ and $0.042 \pm 0.001$ VEM respectively. The best fit values of the $\lambda$ parameters are displayed in the right panel of Fig. 8 as a function of threshold and compared with the best fit values of the $\varphi$ parameters in the left panel. Above a threshold of 2, electrons do no longer contribute. The sharp decrease of the $\lambda$ parameters as a function of threshold illustrates the difficulty of the measurement: the evaluation of the electron charge distribution rests fully on the low threshold data; the higher threshold data are only good at fixing the muon charge distribution.
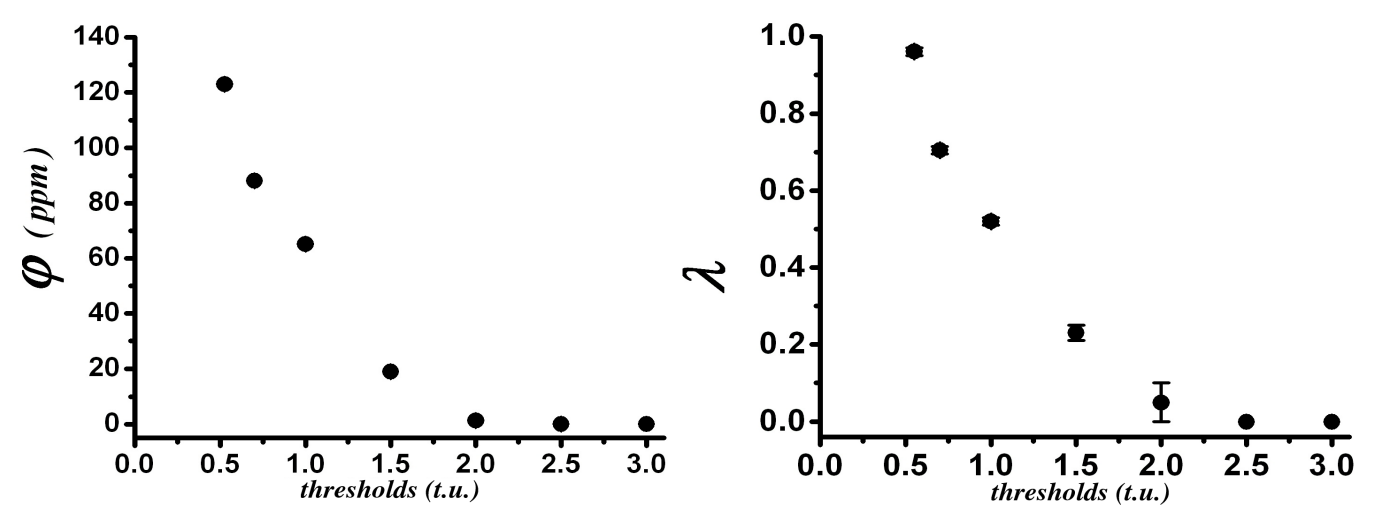

Fig. 8. Dependence on threshold of the $\varphi$ (left panel) and $\lambda$ (right panel) parameters.

The quality of the fits is not excellent, in particular at low charges, and reveals a number of weaknesses of the simple model used in the simulation. Fig. 9 displays the best fit muon distribution $F^{\mu}$ and electron distribution $F^{e l}$ and compares them with the data.
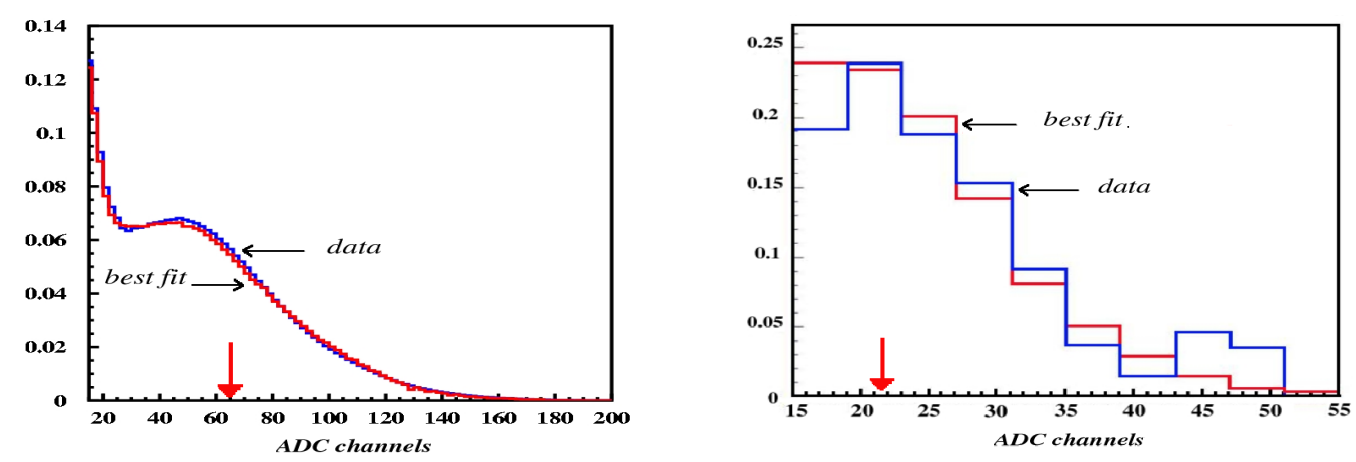

Fig. 9. Muon (left) and electron (right) charge distributions: the best fits $F^{\mu}$ and $F^{e l}$ are shown in red and the data in blue. The arrows indicate the VEM value (left) and one third of it (right). 


\section{SIMULATION AND INTERPRETATION OF THE RESULTS}

The results presented above are now compared with the prediction of a simulation of the detection process taking into account the weaknesses revealed by the analysis of the previous section. As the contribution of muon pairs from a same shower can be neglected, we consider only two kinds of events: either a pair of uncorrelated muons (from two independent showers) or a stopping muon decaying in the water volume.

In both cases, muons are given a kinetic energy $T$ having a Gaussian distribution of adjustable mean $T_{\text {mean }}$, and a zenith angle $\theta$ having a distribution [5] $d N / d \theta=N_{0} \cos ^{2} \theta(1-$ $0.108 \sin ^{2} \theta$ ) between $0^{\circ}$ and $90^{\circ}$. The choice of a Gaussian rather than exponential kinetic energy distribution corresponds to available measurements [7] in the low energy range $(T<\sim 20 \mathrm{GeV})$. Here, $N$ is the muon flux per unit of solid angle, of area (normal to the trajectory) and of time. The charge is calculated in VEM using a Poisson distribution of photoelectrons, the mean number of photoelectrons per VEM being an adjustable parameter, $\nu$.

The effect of the threshold $k_{t h r}$ (measured in threshold units) on a charge $q$ is simulated separately on each of the two phototube signals that are summed to produce the analysed charge. As both phototube signals are not strongly correlated, even if the cut-offs were sharp, which they are not, the resulting cut-off on the sum signal would be far from sharp. Overlooking this feature causes bad fits in the low charge region: the effect of the discriminator thresholds cannot be simulated by a simple cut-off on the sum signal.

A main weakness of the model used in the previous section is made clear by comparing the muon charge distributions of Fig. 4 right and Fig. 9 left, showing that the muon charge distribution, including or not stopping muons, is not expected to peak at low charges while the data require a muon contribution that does. Indeed, a low charge component, the so-called soft component has been known for many years [8] and is essentially composed of soft electrons, positrons and photons. As it is not penetrating, it does not show up whenever a coincidence between two different detectors is required; however, in the present case where the coincidence is between two PMTs looking at the same water volume, there is no such suppression. It is also present in the PAO data [9], however at a different rate because of the different altitude (1400 $\mathrm{m}$ rather than sea level). In order to include such a soft component in the simulation, we use an exponential dependence on charge $q$ of the form $d N / d q=q_{\text {soft }}^{-1} \exp \left(-q / q_{\text {soft }}\right)$ where $q_{\text {soft }}$ is an adjustable parameter. We use as a second adjustable parameter the fraction $f_{\text {soft }}$ of the inclusive rate taken by the soft component.

Muons in muon pair events are separated by a time $t$ uniformly distributed between 0 and the width of the time window, $10 \mu \mathrm{s}$. So does also the soft component.

Muon decays are simulated by generating parent muons having a track length $l$ in excess of $11 \mathrm{~cm}$, below which a stopping muon does not emit any Cherenkov light. The position $x_{\text {stop }}$ of the stop on the track is taken with a uniform distribution between the track exit and a point shifted by $11 \mathrm{~cm}$ from the entrance end inside the water volume, each value of $x_{\text {stop }}$ being given a weight accounting for its likelihood, namely $d N / d x_{\text {stop }}=(d N / d E) /\left(d x_{\text {stop }} / d E\right)$.

The charge of the PMT signals associated with the Cherenkov light emitted by an electron shower of energy $E_{\text {decay }}$ is averaged over the electron energy and direction; it is taken to correspond to an energy smaller than $E_{\text {decay }}$ by a factor accounting for the 
possibly incomplete containment of the shower, measured by an adjustable shower size parameter $\Lambda$. The adjustable parameter $E_{\text {decay }}=x E_{\text {end }}$ is taken with a standard muon decay distribution $\left(d N / d x=6 x^{2}-4 x^{3}\right)$ having its end point $(x=1)$ at $E_{\text {end }}$ where $E_{\text {end }}$ is an adjustable parameter measured in VEM.

The muon decay time distribution is taken exponential with an effective decay time of $2.0 \mu \mathrm{s}$ accounting, on average, for muon capture in water and the decay electron is required to be emitted within the accepted window (width $W_{1}$ and delay $D_{1}$ ).

We have simulated the mechanism of light collection with the aim of revealing a possible dependence on zenith angle of its efficiency. We simulated both Lambertian diffusion on the tank walls (as is probably the case for the PAO where the walls are made of Tyvek) and specular reflection (expected to contribute in the VATLY case where the walls are coated with aluminized Mylar). We define a light attenuation length in water, $\Lambda_{\text {att }}$ and a diffusion (or reflection) coefficient $\eta$ describing the ratio between the diffused (or reflected) and incident light on wall encounters, using as default values: $\Lambda_{a t t}=20 \mathrm{~m}$ and $\eta=0.85$.

In the case of a perfect optical cavity, $\Lambda_{\text {att }}=\infty$ and $\eta=1$, any Cherenkov photon emitted along a muon track ultimately escapes into one of the PMTs. However, the average number of reflections or diffusions it takes to achieve this, may be zenith angle dependent. Similarly, the time it takes, i.e. the optical path length, may also be zenith angle dependent. Namely, in a perfect optical cavity, one expects the area of the pulse shapes to be zenith angle independent but not their widths. In a real optical cavity, $\Lambda_{\text {att }}$ takes a finite value and $\eta$ is smaller than unity. The detected signal becomes $N \eta^{k} \exp \left(-l / \Lambda_{a t t}\right)$ where $N$ is the number of Cherenkov photons emitted, $k$ the number of wall reflections (or diffusions) and $l$ the optical path that precede the escape into the PMT photocathode.
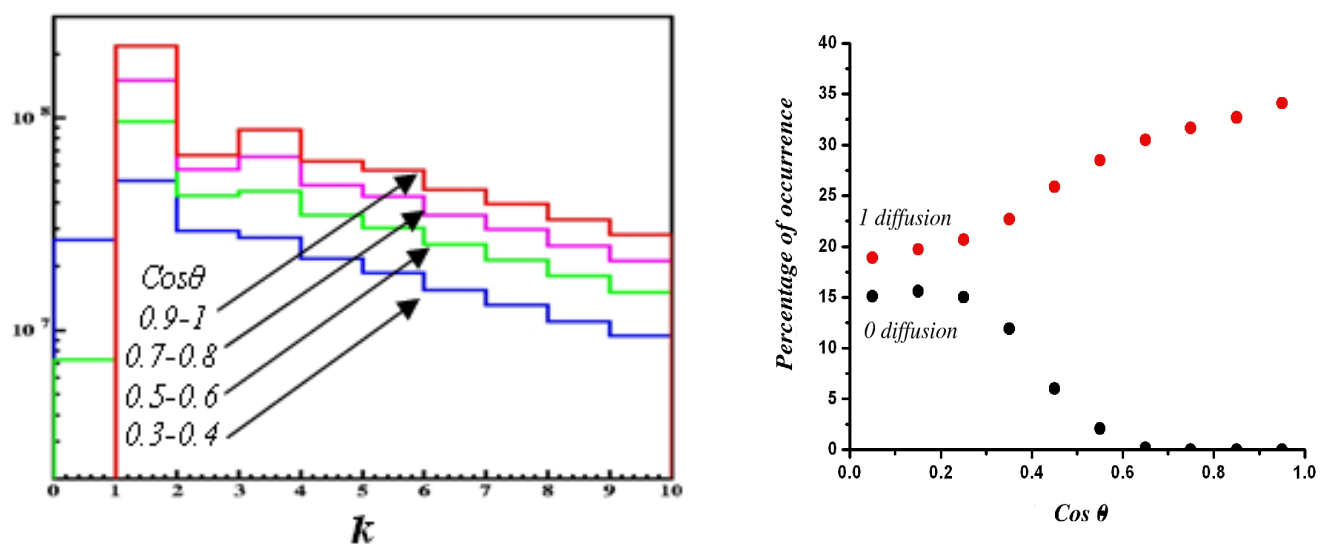

Fig. 10. Left: distribution of the number of diffusions preceding detection by the PMTs for $\cos \theta=0.3$ to $0.4,0.5$ to $0.6,0.7$ to 0.8 and 0.9 to 1 . Right: Relative occurrence $(\%)$ of respectively zero and one diffusions preceding detection by the PMTs as a function of $\cos \theta$.

An effect that produces a dependence of the light collection efficiency over zenith angle is the existence of direct light. It results from the fact that it becomes possible for Cherenkov light to reach the PMTs without any diffusion or reflection when the zenith 
angle exceeds $41^{\circ}(\cos \theta=0.75)$. Fig. 10 displays the distribution of the number of diffusions or reflections that occur before reaching the PMTs for different intervals of $\cos \theta$. It shows clearly how for small zenith angles direct light (no preceding diffusion or reflection) is relatively suppressed, while it becomes more and more important when the zenith angle increases.

In general, in the case of a non-perfect optical cavity, one may then expect a zenith angle dependence of the light collection efficiency. However, to the extent that the number of photons effectively collected in the PMTs is much smaller than the total number of Cherenkov photons produced, this dependence cannot be very important. Indeed, in such a case, each Cherenkov photon has a small probability $P$, in principle dependent on $\cos \theta$, to be detected after a given optical length $l_{\max }$ and a given number of reflections/diffusions $k_{\max }$. But the average values of $l$ and $k$ are only slightly smaller than $l_{\max } / 2$ and $k_{\max } / 2$ respectively to the extent that only few photons have been collected before reaching $l_{\text {max }}$ or $k_{\max }$. As the effective values of $l_{\max }$ and $k_{\max }$ are defined solely by $\Lambda_{\text {att }}$ and $\eta$, they do not depend on $\cos \theta$. Moreover, as the light collection efficiency is completely defined by the average values taken by $l$ and $k$, it will not depend on $\cos \theta$ either. This is indeed what the simulation predicts: Fig. 11 displays the dependence on zenith angle of the mean number of photoelectrons per VEM for $\Lambda_{a t t}=20 \mathrm{~m}$ and $\eta=0.85$. In such a case, the light is attenuated by a factor 100 after $\sim 28$ reflections/diffusions or after $\sim 92 \mathrm{~m}$ optical path. The dependence on $\cos \theta$ is indeed small, particularly in the case of Lambertian diffusion, the main effect being that of direct light in the case of specular reflection.

For each value of the threshold $T$ and of the time delay $D(\mu \mathrm{s})$, data are compared with the best fit result of the simulation in the appendix. The values obtained for the parameters that have been adjusted are listed in Table 3. Fig. 12 illustrates two typical cases. The first, with $T=0.5$ t.u. and $D=0.5 \mu$ s shows a clear electron contribution at small time values, somewhat contaminated by the soft component. The second, with $T=2.0$ t.u. and $D=5.0 \mu$ s is exclusively populated by muons with no noticeable time dependence. The uncertainties that are quoted neglect correlations between the parameters: they simply correspond to the shift of the parameter with respect to the best fit value such that the $\chi^{2}$ per degree of freedom (of which there are 10199) increases by $1 \%$. Properly speaking, they are therefore rather indicators of the sensitivity of each particular parameter to the quality of the fit. We now comment each of these in turn:

- The number of photoelectrons per VEM is $\nu=13.0 \pm 0.9$ in good agreement with our earlier estimate of 14 obtained from the width of the calibration curves. This number is really an effective number of photoelectrons per VEM, including other effects that might cause a smearing of the charge measurement. It is rewarding to find that the effect is consistently described by a single value in both the VEM region and in the low charge regime (stopping muons and decay electrons).

- The value of the end point of the charge distribution of decay electrons is $E_{\text {end }}=0.275 \pm 0.018$ VEM. We note that it is unnecessary to smear this distribution beyond the natural smearing resulting from photoelectron statistics. The resulting smeared distribution is displayed in Fig. 13. This result is consistent with the value obtained in PAO data, where the mean decay electron charge is 0.12 VEM.

- The soft component is described by $f_{\text {soft }}=0.795 \pm 0.012$ and $q_{\text {soft }}=0.32 \pm 0.02$ VEM. The high value of $f_{\text {soft }}$ is somewhat misleading to the extent that charges smaller 


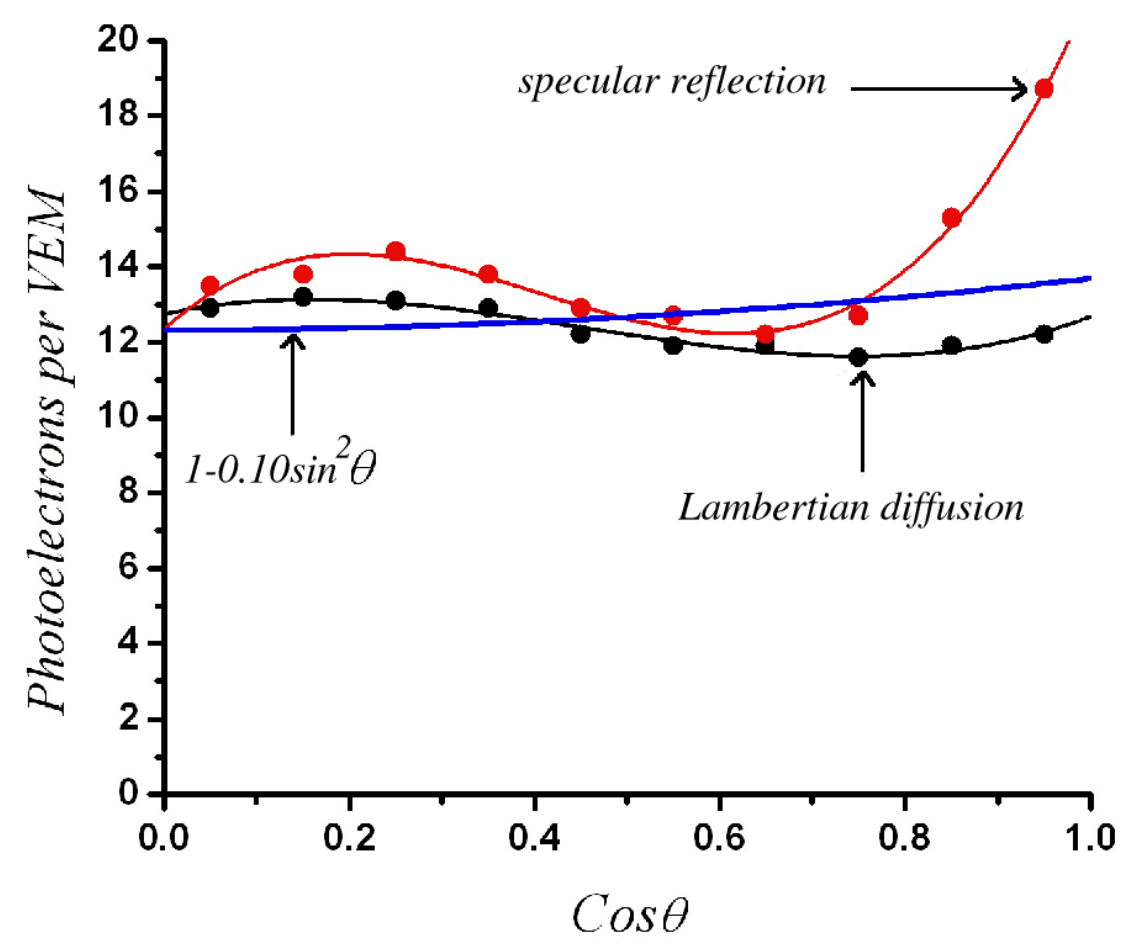

Fig. 11. Dependence on $\cos \theta$ of the mean number of photoelectrons per VEM for $\Lambda_{a t t}=20 \mathrm{~m}$ and $\eta=0.85$ for Lambertian diffusion and specular reflection. A zenith angle dependence of the form $1-0.10 \sin ^{2} \theta$, as required by the best fit, is also shown.

than 0.1 VEM are cut by the threshold. Indeed, Fig. 13 displays the soft component in the range where it is observed and where it can be compared with the electron and muon contributions.

- The value taken by $\Lambda, 36 \pm 6 \mathrm{~cm}$, is (by chance) precisely equal to the value of the radiation length in water, however with a large error; indeed, this parameter is only an ad hoc way to simulate the fiducial volume effect and there is no reason for it to be precisely equal to the radiation length although it is expected to be of the same order of magnitude.

- The parameters describing the dependence of the cut-off function on $k_{t h r}$ are $a_{t h r}=0.022 \pm 0.002 \mathrm{VEM}, b_{t h r}=0.0495 \pm 0.0013 \mathrm{VEM}$ per threshold unit and $c_{t h r}=0.035 \pm 0.006$ VEM per threshold unit. The value of $a_{t h r}$ is an order of magnitude smaller than obtained in Section IV.2 because it now applies to each PMT signal separately rather than to their average value. The value of $c_{t h r}$ deviates significantly from zero, although most of the smearing effect is naturally produced by the mechanism described earlier.

- The mean muon kinetic energy is $E_{\text {mean }}=4.0_{-0.3}^{+0.4} \mathrm{GeV}$, in excellent agreement with 

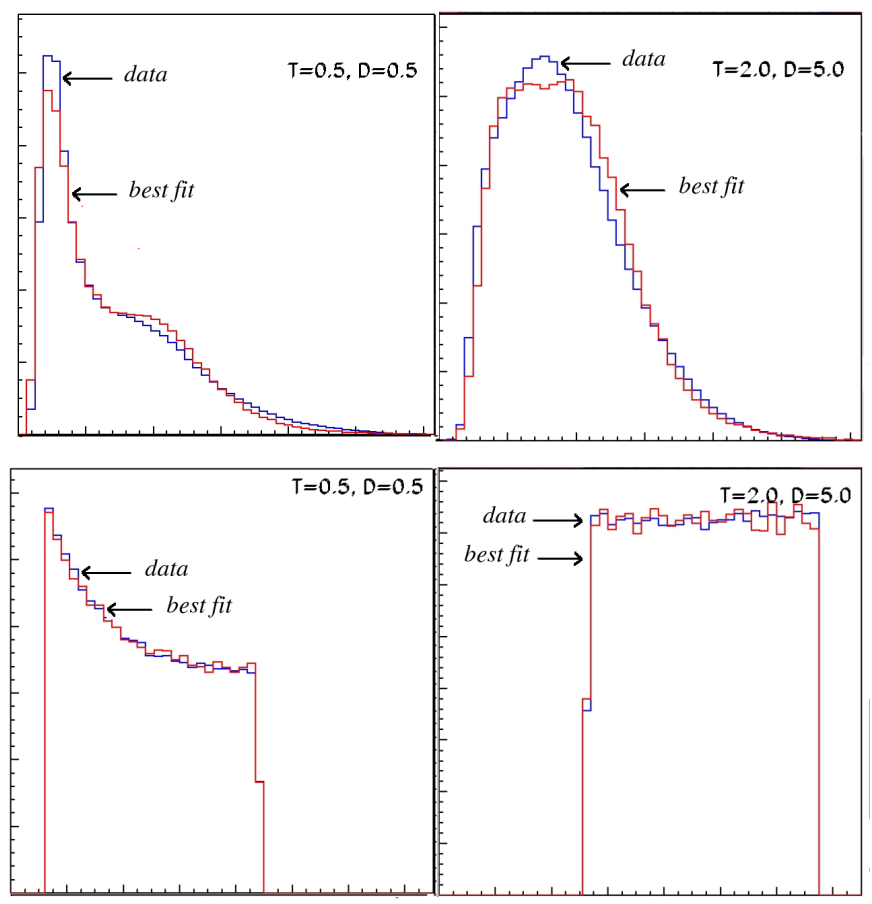

Fig. 12. Best fit results of the simulation are compared with data for both charge (upper panels, 3.08 VEM full scale) and time (lower panel, $15 \mu$ s full scale) distribution. The left panels are for $T=0.5 \mathrm{t} . u$. and $D=0.5 \mu \mathrm{s}$ and the right panel for $T=2.0$ t.u. and $D=5.0 \mu \mathrm{s}$.

Table 3. Best fit values of the model parameters.

\begin{tabular}{|c|c|c|}
\hline Parameter & Symbol & Value (error) \\
\hline Soft component probability & $f_{\text {soft }}$ & $0.795(0.012)$ \\
\hline Soft component width (VEM) & $q_{\text {soft }}$ & $0.32(0.02)$ \\
\hline Decay electron end point(VEM) & $E_{\text {end }}$ & $0.275(0.018)$ \\
\hline Shower size (cm) & $\Lambda$ & $36(6)$ \\
\hline Mean muon kinetic energy (GeV) & $E_{\text {mean }}$ & $4.0\left(_{-0.3}^{+0.4}\right)$ \\
\hline Number of photoelectrons per VEM & $\nu$ & $13.0(0.9)$ \\
\hline Threshold offset (VEM) & $a_{t h r}$ & $0.022(0.002)$ \\
\hline Cut-off slope (VEM/t.u) & $b_{t h r}$ & $0.0495(0.0013)$ \\
\hline Cut-off width (VEM/t.u) & $c_{t h r}$ & $0.035(0.006)$ \\
\hline Light collection efficiency parameter & $\xi$ & $0.10(0.04)$ \\
\hline
\end{tabular}

the expected value [7]. It is remarkable that the data are able to measure it properly in such an indirect way.

- Assuming that the optical properties of the tank are better described by a Lambertian diffusion than by a specular reflection (although, as already mentioned, we expect 
an intermediate situation), the fit was performed by neglecting a possible dependence of the light collection efficiency on zenith angle. Including a dependence on zenith angle of the form $1-\xi \sin ^{2} \theta$ predicts a value $\xi=0.10 \pm 0.04$, in good agreement with the analysis performed earlier (Fig. 11) and suggesting that $\Lambda_{a t t}=20 \mathrm{~m}$ and $\eta=0.85$ are indeed sensible estimates of the optical quality of the tank cavity.

Fig. 13 displays the respective contributions of the soft component, muons and decay electrons to the charge distribution at low threshold and for both a small and a large value of the delay. It illustrates the difficulty of the measurement, the decay electron component becoming negligible for charges in excess of $\sim 0.5 \mathrm{VEM}$, and being largely hidden behind the soft component. Fig. 14 displays the charge distribution associated with Cherenkov photons emitted by stopping muons that produce detected decay electrons. The figure is drawn for the lowest threshold value and a delay $D_{1}=0.5 \mu \mathrm{s}$. Its shape is nearly the same for a delay of $5 \mu \mathrm{s}$ (but its amplitude is of course much smaller). The mean value of the charge distribution displayed in Fig. 14 is 0.54 VEM. Such a small value, although larger than that of the electron distribution, adds to the difficulty to detect electrons from muon decays when using a Cherenkov detector.
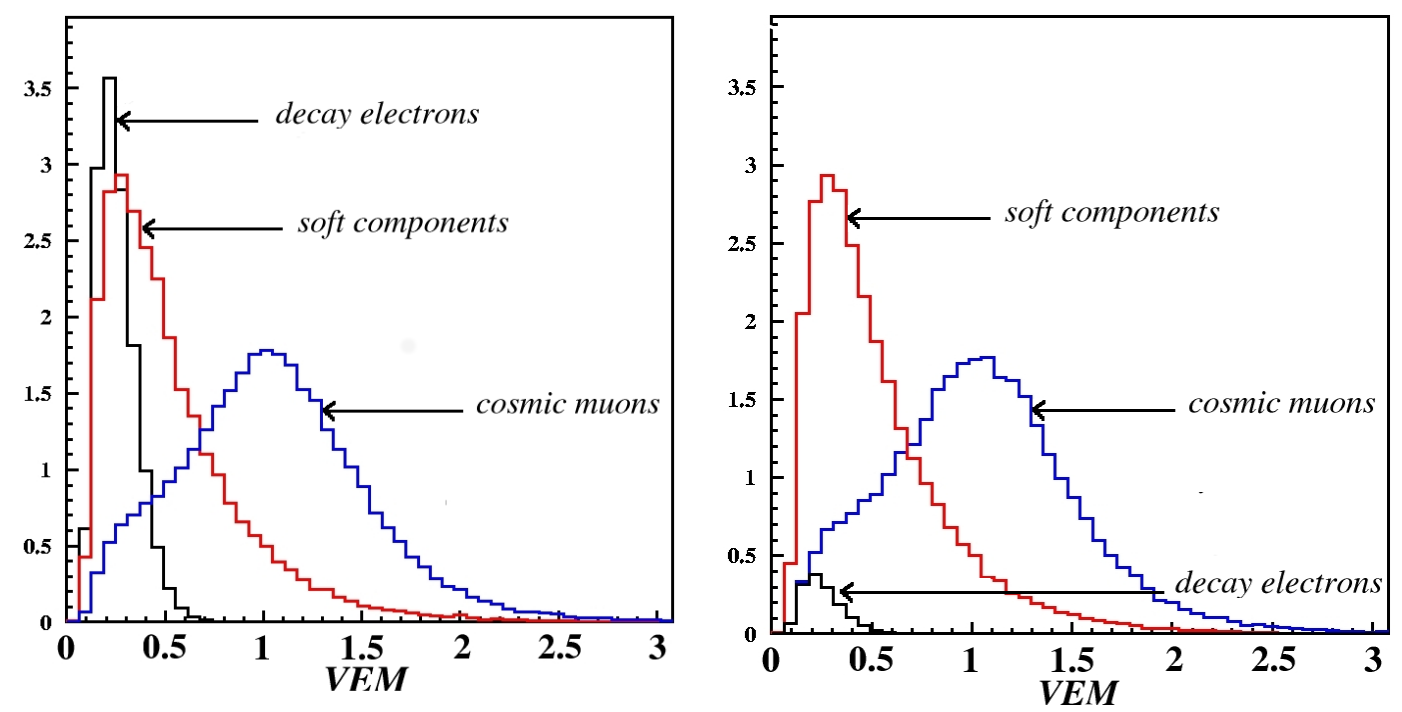

Fig. 13. Respective contributions of the soft component, decay electrons and cosmic muons for the smallest threshold value ( 0.5 threshold units) and respective delays of $0.5 \mu \mathrm{s}$ (left) and $5.0 \mu \mathrm{s}$ (right).

\section{SUMMARY}

We have collected a large sample of data that provide very clear evidence for muon decays with the expected time dependence including a small contribution from muon capture in oxygen. The amplitude of the electron signal is observed at the level of a fraction of a VEM, and only the upper part of its distribution can be detected. Its end point is measured to be $0.275 \pm 0.018 \mathrm{VEM}$ in agreement with the PAO value of a mean charge of 


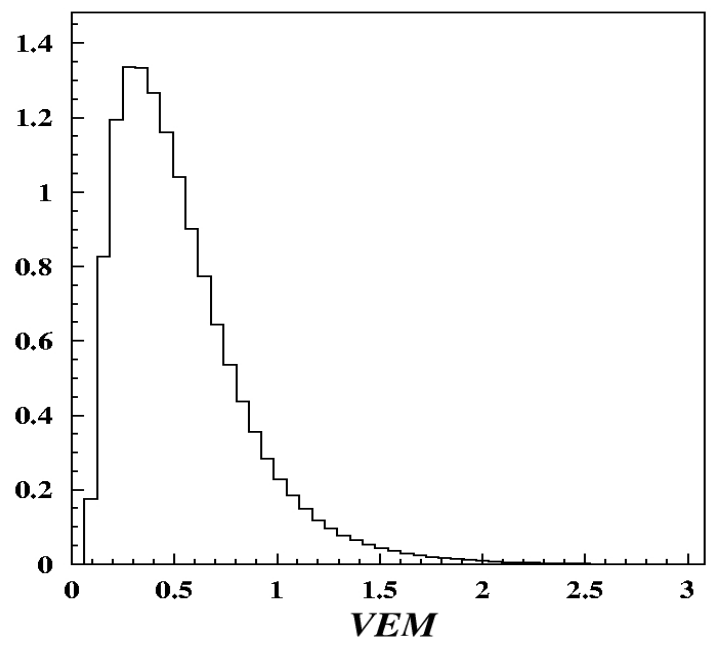

Fig. 14. Charge distribution (VEM) associated with stopping muons that produce a detected decay electron for a threshold of 0.5 threshold units and a delay $D_{1}$ of $0.5 \mu \mathrm{s}$.

0.12 VEM. The muon distribution provides evidence for a soft component, known to be essentially made of electrons, positrons and photons, which appears particularly important in the present experimental set-up due to the large sensitive volume of the Cherenkov detector. Good fits of the measured data to a simple model of the detection process have been obtained for both the charge and time distributions. They allow obtaining useful evaluations of the number of photoelectrons per VEM, $13.0 \pm 0.9$ and of the mean muon energy, $4.0_{-0.3}^{+0.4} \mathrm{GeV}$. The detection efficiency of electrons has been modelled using an estimate of the effective electron shower size found at the scale of the radiation length in water $(36 \mathrm{~cm})$ as expected. The occurrence of muon pairs from a same shower has been measured with a rate of $7.0 \pm 0.5 \mathrm{~Hz}$, implying a decoherence function of the order of $30 \mathrm{~m}$ for a sea level multiplicity of two muons per shower.

The availability of a replica of a PAO Cherenkov detector in our laboratory has proven to be useful not only for training purposes but also for contributing a better understanding of the response of such a detector, in particular to low amplitude signals at the level of a fraction of a VEM. It will continue to be used as a training tool for students, not only at the scale of the VATLY team but at a broader scale.

\section{ACKNOWLEDGMENTS}

We are indebted to the Pierre Auger Collaboration for their constant interest and support. Financial support is acknowledged from the National Foundation for Science \& Technology Development (NAFOSTED), from the Institute for Nuclear Science and Technology, from the World Laboratory, from the Odon Vallet fellowships, from the French CNRS and from the Rencontres du Vietnam.

This work was done in partial fulfilment of the $\mathrm{PhD}$ degree of one of the authors (N.T.Thao). 


\section{REFERENCES}

[1] The Pierre Auger Collaboration, Nucl. Inst. Meth. A 523 (2004) 50 and references therein.

[2] The Pierre Auger Collaboration, Contributions to the 32nd International Cosmic Ray Conference, Beijing 2011, and references therein.

[3] J. Abraham et al. (Auger Collaboration), Phys. Lett. B 685 (2010) 239-246; M. Settimo et al. (Auger Collaboration), Eur. Phys. J. Plus 127 (2012) 87.

[4] J. Abraham et al. (Auger Collaboration), JCAP 04 (2012) 040; J. Abraham et al. (Auger Collaboration), ApJL $\mathbf{7 6 2}$ (2012) L13.

[5] P.N. Dinh et al., Measurement of the vertical cosmic muon flux in a region of large rigidity cut-off, $N u c l$. Phys. B 627 (2002) 29-42;

P. N. Dinh, et al., Nucl. Phys. B 661 (2003) 3-16;

P. N. Diep, et al., Nucl. Phys. B 678 (2004) 3-15;

N. T. Thao, The Detection of Extensive Air Showers in Hanoi, Master thesis presented to the Hanoi University of Sciences, Vietnam National University, 2007;

P. T. T. Nhung, Performance Studies of Water Cherenkov Counters, Master thesis presented to the Hanoi University of Sciences, Vietnam National University, 2006;

P. N. Dong, The Cherenkov Counters of the VATLY Laboratory, Master thesis presented to the Hanoi University of Technology, Vietnam National University, 2006;

D. T. The, Optical properties of a Cherenkov counter, Diploma in Astrophysics, Hanoi University of Education, Hanoi, May 2007;

P. N. Diep, Study of the Effects of Atmospheric Pressure on the Cosmic Muon Rate in Ha Noi, Diploma in Physics, Ha Noi National University of Sciences, 2003;

P.T.T. Nhung, Study of the Effects of Temperature on the Cosmic Muon Rate in Ha Noi, Diploma in Physics, Ha Noi National University of Sciences, 2003.

[6] The PDG Group, Particle Physics Booklet, 2008, p. 220.

[7] M. Honda et al., Phys. Rev. D 52 (1995) 4985 and Proc. 2001 Int. Cosmic Ray Conf., Copernicus Gesellschaft, Hamburg, p. 1162.

[8] Y. Nishina, M. Takeuchi and T. Ichimiya, Phys. Rev. 52 (1937) 1198.

[9] P. N. Diep, Contribution to the Identification of Primary Ultra High Energy Cosmic Rays Using the Pierre Auger Observatory, $\mathrm{PhD}$ thesis, 2010, and references therein.

\section{APPENDIX}

The figures below compare the charge and time data with the results of the simulation. Each panel is labelled by the values taken by the threshold $T$ (in threshold units) and by the time delay $D$ (in microseconds). Electrons and soft component contribute to the low threshold data exclusively and, for these, the electron contribution decreases with the time delay while that of the soft component remains constant. 
N. T. THAO, P. N. DIEP, P. N. DONG, P. T. T. NHUNG, P. DARRIUlAT, P. T. ANH, AND D. T. HOAI 259

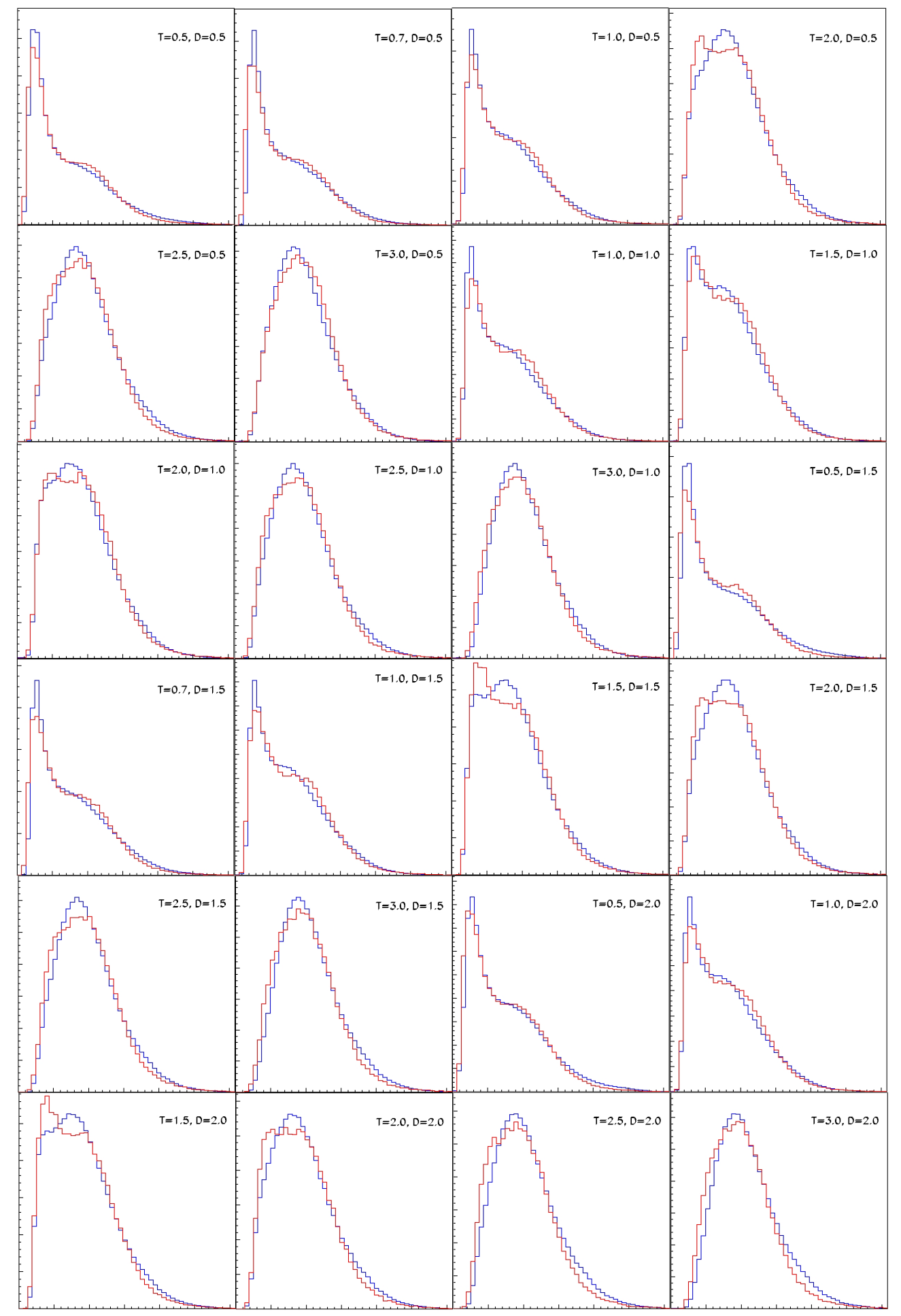




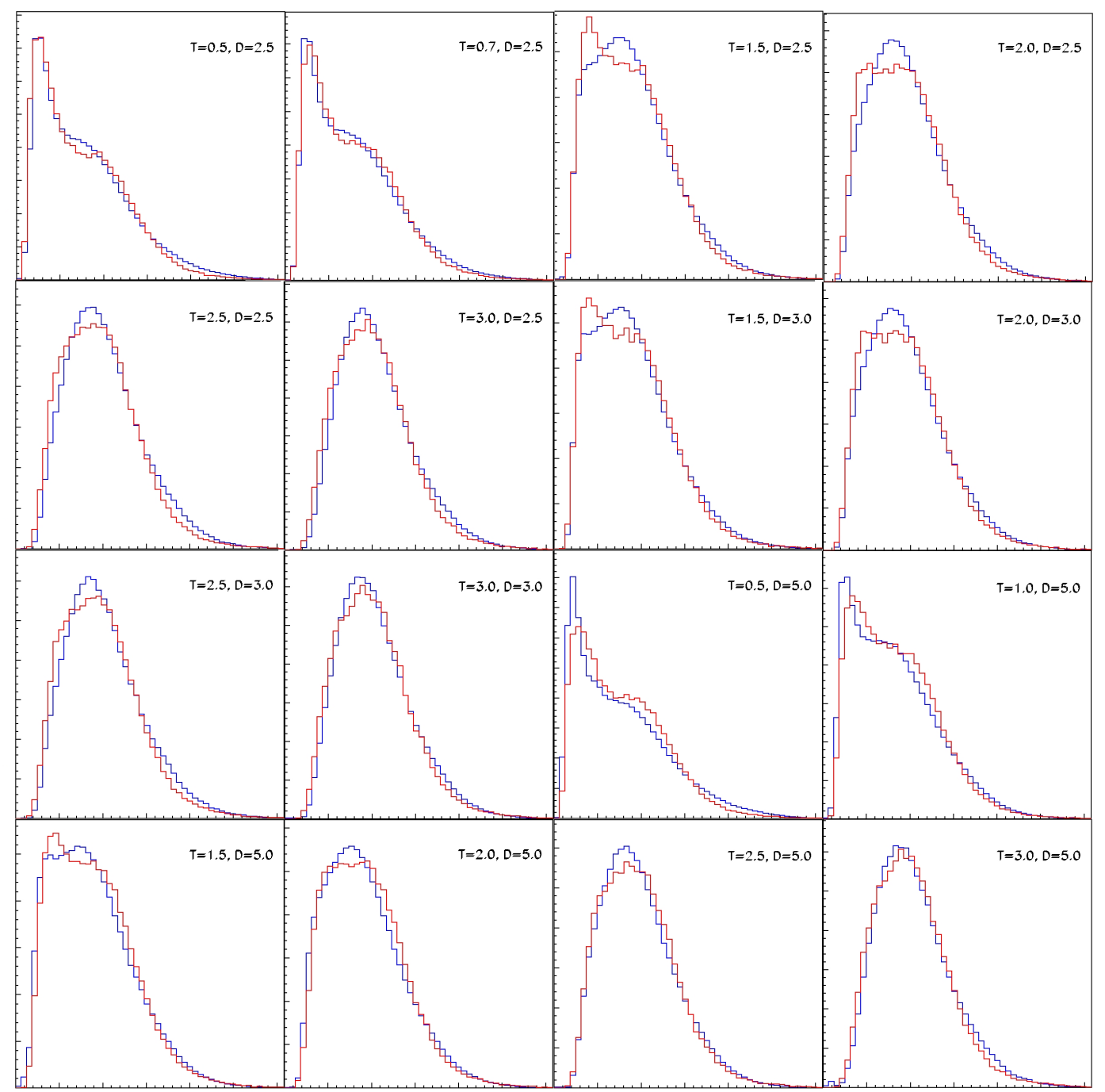

Fig. 15. Charge distributions measured and predicted for different delays and thresholds. The full scale is $3.08 \mathrm{VEM}$ on each panel. Each panel is labeled by its threshold $T$ (in threshold units) and its delay $D$ (in microseconds). 
N. T. THAO, P. N. DIEP, P. N. DONG, P. T. T. NHUNG, P. DARRIUlAT, P. T. ANH, AND D. T. HOAI 261

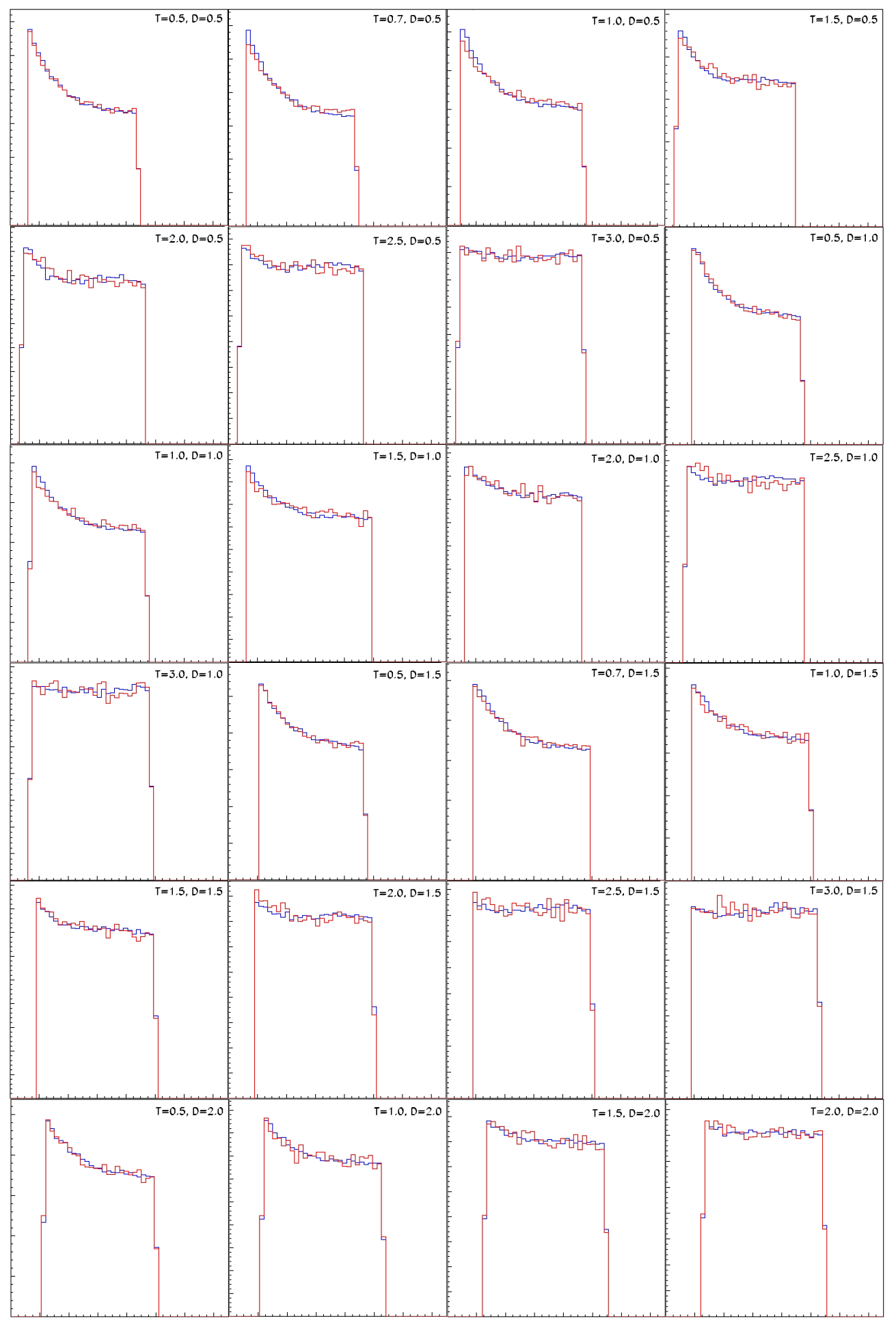




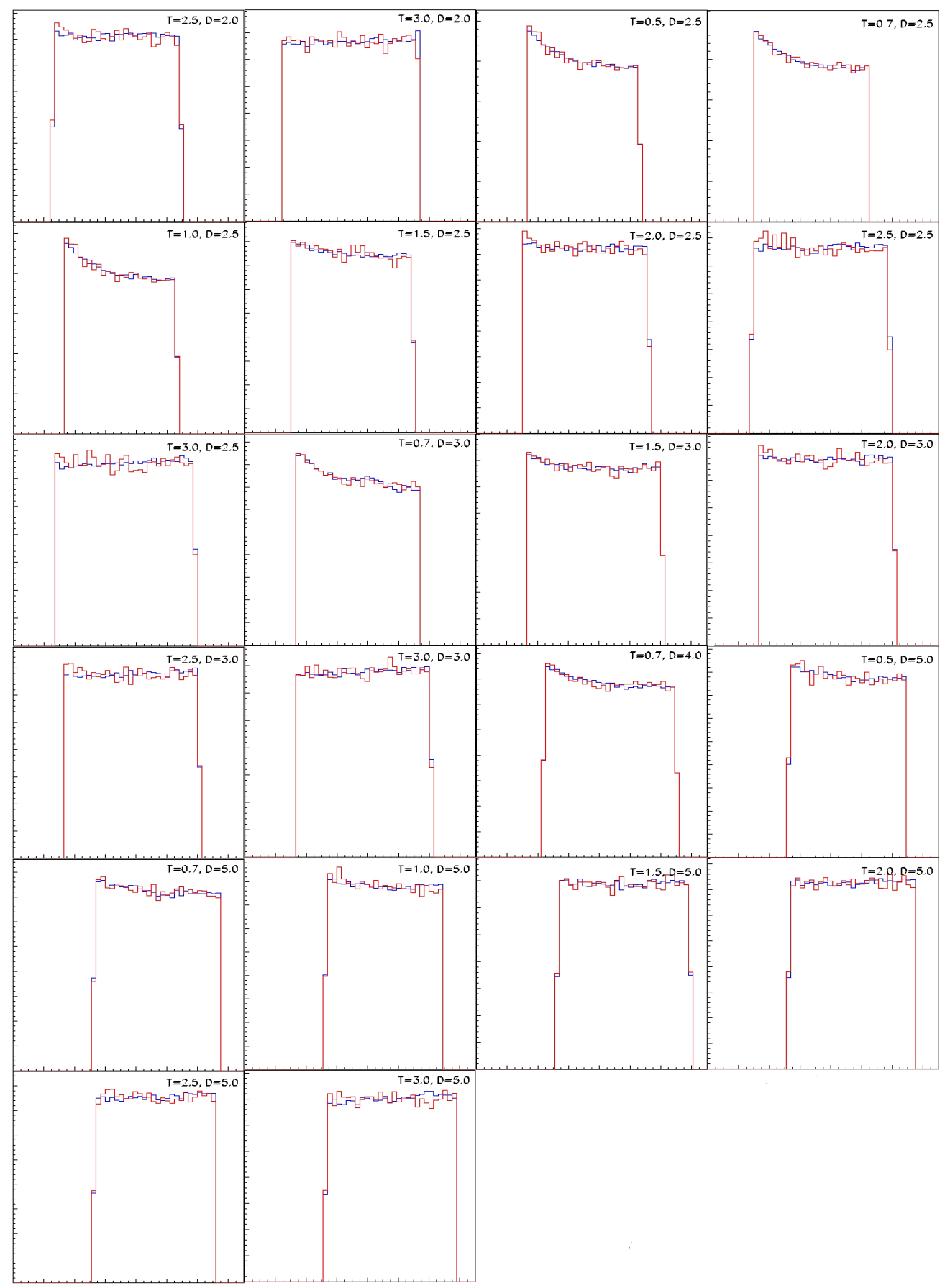

Fig. 16. Time distributions measured and predicted. The full scale is $15 \mu \mathrm{s}$ on each panel. Each panel is labeled by its threshold $T$ (in threshold units) and its delay $D$ (in microseconds). 